\title{
Earth and space-based NEO survey simulations: prospects for achieving the Spaceguard Goal
}

\author{
Robert Jedicke, ${ }^{\mathrm{a}, *}$ Alessandro Morbidelli, ${ }^{\mathrm{b}}$ Timothy Spahr, ${ }^{\mathrm{c}}$ Jean-Marc Petit, ${ }^{\mathrm{b}}$ \\ and William F. Bottke Jr. ${ }^{\text {d }}$ \\ ${ }^{a}$ Lunar and Planetary Laboratory, University of Arizona, Tucson, AZ 85721, USA \\ ${ }^{\mathrm{b}}$ Obs. de la Cote d'Azur, B.P. 4229, 06034 Nice Cedex 4, France \\ ${ }^{\mathrm{c}}$ Smithsonian Astrophysical Observatory, 60 Garden Street, Cambridge, MA 02138, USA \\ ${ }^{\mathrm{d}}$ Southwest Research Institute, 1050 Walnut Street, Suite 426, Boulder, CO 80302, USA
}

Received 20 December 2001; revised 3 September 2002

\begin{abstract}
We have used an improved model of the orbit and absolute magnitude distribution of Near Earth Objects (NEOs) to simulate the performance of asteroid surveys. Our results support general conclusions of previous studies using preliminary Near Earth Asteroid (NEA) orbit and magnitude distributions and suggest that meeting the Spaceguard Goal of $90 \%$ completion for Near Earth Objects (NEOs) greater than $1 \mathrm{~km}$ diameter by 2008 is impossible given contemporary surveying capabilities.

The NEO model was derived from NEO detections by the Spacewatch Project. For this paper we developed a simulator for the Catalina Sky Survey (CSS) for which we had a complete pointing history and NEO detection efficiency. The good match between the output of the simulator and the actual CSS performance gives confidence that both the NEO model and simulator are correct. Then, in order to determine if existing surveys can meet the Spaceguard Goal, we developed a simulator to mimic the LINEAR survey, for which detailed performance characteristics were unavailable. This simulator serendipitously provided an estimate for the currently undiscovered population of NEOs upon which we base all our estimates of time to $90 \%$ completion. We also developed a set of idealized NEO surveys in order to constrain the best possible survey performance in contrast to more realistic systems.

A $100 \%$ efficient, all-sky, every night survey, subject only to the constraints of detection above a specified air mass and when the Sun is $18^{\circ}$ below the horizon provides a benchmark from which to examine the effect of imposing more restrictions and the efficacy of some simple survey strategies. Such a survey must have a limiting V-magnitude of $20.1 \pm 0.2$ to meet the Spaceguard Goal.

More realistic surveys, limited by latitude, the galaxy, minimum rates of NEO motion, etc., require fainter limiting magnitudes to reach the same completion. Our most realistic simulations, which have been normalized to the performance of the LINEAR detector system's operation in the period 1999-2000, indicate that it would take them another $33 \pm 5$ years to reach $90 \%$ completeness for the larger asteroids ( $\gtrsim 1 \mathrm{~km}$ diameter). They would need to immediately increase the limiting magnitude to about 24 in order to meet the Spaceguard Goal.

The simulations suggest that there may be little need for distributing survey telescopes in longitude and latitude as long as there is sufficient sky coverage from a telescope or network of telescopes which may be geographically close. An idealized space-based survey, especially from a satellite orbit much interior to Earth, would offer an advantage over their terrestrial counterparts. We do not consider a cost-benefit analysis for any of the simulations but suspect that a local-area network of telescopes capable of covering much of the sky in a month to $V \sim 21.5$ may be administratively, financially, and scientifically the best compromise for reaching $90 \%$ completion of NEOs larger than $1 \mathrm{~km}$ diameter.
\end{abstract}

(C) 2003 Elsevier Science (USA). All rights reserved.

Keyword: Asteroids

* Corresponding author. Fax: +520-621-4933.

E-mail address: jedicke@pirl.lpl.arizona.edu (R. Jedicke). 


\section{Introduction}

The realization that globally devastating asteroid or comet impacts occur on time scales relevant to human beings has recently spurred the world into developing survey systems capable of detecting Near Earth Objects (NEOs) which threaten Earth. (A NEO is an asteroidal or cometary body with a perihelion distance $q \leq 1.3 \mathrm{AU}$ and aphelion distance $Q \geq 0.983$ AU.) This hazard is one of the few worldwide dangers which may be almost fully quantified within the next few decades. The only caveat is the relatively small risk (Levison et al., 2002) due to long period comets (LPC), which are unlikely to have been seen before and therefore cannot be predicted. In this paper we examine the prospects for discovering $90 \%$ of all NEOs with absolute magnitude $H \leq 18$ by 2008. (In May 1998 NASA committed to achieving this level of completion within ten years. This target will hereafter be referred to as the 'Spaceguard Goal.' See Morrison et al., 1992)

Our main motivation is to determine whether existing NEO surveys can meet the Spaceguard Goal. If they cannot meet this target then it is important to ask to what depth the surveys must scan the sky to achieve the goal in a reasonable time frame? What is "reasonable"? Simple linear extrapolations of NEO discovery rates to the expected total population predict that the Spaceguard Goal is achievable; but this assumes that the undiscovered NEOs are as likely to be discovered as the known sample. In the process of answering these questions we will also touch upon the benefits of space-based surveys and examine the merits of multihemispheric NEO surveying observatories.

Previous efforts to simulate the discovery of NEOs (e.g., Morrison et al., 1992; Bowell and Muinonen, 1994; Muinonen, 1998; Harris, 1998; Tedesco et al., 2000) used either the known set of NEO orbits or early unbiased estimates of the population. Their set of orbits was passed through a simulation of a NEO survey in order to estimate its efficiency at detecting the NEOs. Some of these simulations incorporated detailed detector and weather modelling or examined the opportunity of determining good orbits for the discovered objects. All of them simulated the surveys starting from initial conditions in which no NEOs were known. Unfortunately, it is incorrect to claim that the length of time to a given NEO completion level can be determined from the figures presented in those analyses. We will consider the results of the previous studies in Section 5.1.

Our study advances the simulation of NEO surveys because it (i) incorporates a new four-dimensional model of the NEO orbit element and absolute magnitude distribution, (ii) includes an analysis of the statistical variation of the survey's performance based on different input NEO models which are all consistent with the possible population, and (iii) is the first to distinguish between the class of known NEOs and those which are currently undiscovered. With this information we are able to predict a lower limit for the time to completion for a NEO survey (as a function of calendar year since we examine the discoveries of only the currently unknown NEOs).

Recent estimates of the NEO population (e.g., Bottke et al., 2000; Rabinowitz et al., 2000; D’Abramo et al., 2001; Stuart, 2001; Bottke et al., 2002) suggest that there are on the order of 1000 with $H \leq 18$-corresponding very roughly to the size at which impacts are expected to cause global devastation (Morrison et al., 1992; Morbidelli et al., 2002). Differences between the number of NEOs presented in those studies is likely due to a lack of consistency in specifying their range of applicability in orbital elements and because different groups choose customized albedos to convert between absolute magnitude $(H)$ and diameter. The only population estimates which provide four-dimensional distributions of NEO orbit elements and absolute magnitude are those developed by Bottke et al., (2002) and Bottke et al. (2000). Their model incorporates NEOs with semimajor axis $a<7.4 \mathrm{AU}$ and all eccentricities $0 \leq e<1$ and inclinations $i<90^{\circ}$ and is valid for $H<22$. It includes the contribution of the Jupiter family comets (JFC) as well as objects whose orbits lie entirely interior to Earth's orbit (IEO).

Bottke et al. (2002) provide a best-fit model for the relative contribution to the NEO region from each of five different source regions (Section 2.1). Their model yields the probability distribution of finding NEOs in $(a, e, i$, $H$ )-(space) which we used to create 15 different NEO populations. Each of the populations corresponds to the probability distribution with slightly different contributions (yet still within the error bars) from each of the five different sources. The models thus represent 15 equally plausible NEO populations and passing each of them through our survey simulators (Section 2.2) provides a measure of the statistical variation of NEO survey performance.

To verify our NEO model and survey simulator we present (Section 3.1) a comparison between its output and the results of the actual Catalina Sky Survey, for which we had full disclosure on pointing history and NEO detection and identification efficiency. Then, in order to explore whether existing surveys can meet the Spaceguard Goal, we developed a simulation to mimic the performance of the LINEAR NEO survey (Stokes et al., 2000). This search program is clearly the dominant existent NEO survey and modeling its performance is thus absolutely required in order to determine if the Spaceguard Goal can be achieved by existing systems. To within the limit of information available on LINEAR's NEO discoveries we showed that we could mimic LINEAR's performance and, serendipitously, we learned that we can create a model for undiscovered NEOs by running our LINEAR simulator on our model NEO population until the simulator discovers as many NEOs as are currently known (Section 4.2). With the model of undiscovered NEOs we then explore the performance of various idealized and pseudo-realistic surveys in Sections 4.3 through 4.6. We summarize with a comparison to pre- 
vious work and discussion of the findings in this paper in Section 5 .

\section{Modelling NEO discovery}

There are two main elements required for simulating the discovery of NEOs: (1) a realistic model of the distribution of their orbital elements and absolute magnitudes and (2) a simulation of the search strategy.

Estimates of the total number and distribution of NEO orbits in semimajor axis $(a)$, eccentricity $(e)$, inclination $(i)$, and absolute magnitude $(H)$ are notoriously difficult to obtain. It is possible to use the set of known NEO orbit elements as input to the survey simulation but this would bias the simulated discoveries because the set of known objects are, almost tautologically, those which are easiest to find. Previous determinations of these distributions (e.g., Rabinowitz et al., 2000; D'Abramo et al., 2001; Stuart, 2001) have been based on correcting the discovery statistics of NEO surveys for observational selection effects. The difficulty with these distributions is that they tend to be one-dimensional projections (in $a, e, i$, and $H$ separately) and are limited in their range by the sensitivity of the surveying systems upon which they are based. To avoid these difficulties we used the model of Bottke et al. (2002), which is described in some detail below.

Simulating the search strategy and discovery of NEOs is relatively straightforward. An ephemeris is generated for every NEO in the model which is then tested for detection by the simulated survey. Our simulator is described in Section 2.2.

\subsection{NEO Model}

We used the model of Bottke et al. (2002) since they provide a 4-dimensional $(a, e, i, H)$ "map" of the actual NEO orbit and absolute magnitude distribution. The model incorporates NEOs derived from five source regions: the $v_{6}$ $(37 \pm 8 \%)$ and $3: 1(25 \pm 3 \%)$ main-belt resonances; the intermediate Mars crossing (IMC) region ( $23 \pm 8 \%$ ), which is resupplied by a forest of Mars and three-body resonances located in the main belt, the outer main-belt $(8 \pm 1 \%)$, and the JFCs $(6 \pm 4 \%)$. In each case the percentage value reported for the source is its contribution to the current NEO population. Each of the source regions is assumed to feed objects into the NEO region at a steady rate. The steadystate distribution of evolved orbits out of each source region has been established through dynamical evolution of objects started in each source. So creating a model NEO population is tantamount to mixing different contributions from each source subject to the constraint that the total number of objects in the model equals the expected population number.

We generated 15 independent sets of 961 NEOs and IEOs with $H \leq 18$ and Tisserand parameter $T_{J}>2$ accord- ing to the model. $T_{J}$ is the pseudo-energy of the Jacobi integral that must be conserved in the restricted circular three-body problem (Sun-Jupiter-comet) (Kresak, 1979),

$$
T_{J}=\frac{a_{J}}{a}+2 \cos i \sqrt{\left(1-e^{2}\right) \frac{a}{a_{J}}},
$$

where $a_{J}$ is the semimajor axis of Jupiter. At $i=0^{\circ}, T_{J}>$ 2 corresponds to $a \sim 8.0 \mathrm{AU}$. There are NEOs with $T_{J} \leq 2$ but these objects are not incorporated into the Bottke et al. (2002) model. Their exclusion may be justified by the work of Levison (2002) (SOM VII) who have shown that the Earth-impact risk due to these comets is about three orders of magnitude less than the risk due to objects already incorporated in the Bottke et al. (2002) model.

Each of the 15 NEO models were generated with different contributions from each of the five source regions consistent with the statistical errors in the Bottke et al. (2002) NEO model. Individual NEOs $(a, e, i, H)$ in each of the models were generated according to the expected distribution for that particular model. In this way our ensemble of models samples both the statistical variation of the models and the statistical variation within the models themselves. The three angular elements for the orbit were generated randomly in the interval $[0,2 \pi)$. The slope parameter $(G)$ was fixed at 0.22 which is the number-weighted mean between bright $(G=0.25)$ and dark $(G=0.15)$ NEOs according to Morbidelli et al. (2002).

\subsection{Survey simulator}

Each of the 15 sets of generated NEOs were run through our survey simulator in various configurations. The simulator is designed to mimic the behavior of a telescopic survey (with preselected characteristics) that searches the night sky for asteroids and comets. To run the code we input a set of objects that includes (i) their Keplerian orbital elements, (ii) their absolute magnitude, and (iii) slope parameter. We also input characteristics corresponding to the nature of the survey under consideration, e.g., the fields of sky it covers at a given time and the limiting magnitude of the system. In some cases the survey might cover the entire sky at one instant while in others it might observe many small rectangular fields during the course of a night. Similarly, the limiting magnitude of the system may be abrupt $(100 \%$ to some magnitude and then $0 \%)$ or gentle $(100 \%$ to some magnitude and then decreasing linearly to $0 \%$ in a fixed range of magnitudes). The position of objects on the sky at any time is provided by an ephemeris calculator-we use routines from the slalib package (http://www.hartrao.ac.za/ nccsdoc/slalib/).

If an object lies in a survey field the code calculates the object's magnitude and the detection efficiency $(\varepsilon)$ at that magnitude in order determine whether a detection is possible. An object is considered detected if a random number $r$ in the range $[0,1)$ is such that $r \leq \varepsilon$. When an object is 
detected for the first time the simulator outputs a list of discovery characteristics (e.g., rates of motion, apparent magnitude, location with respect to opposition). If the object is detected but has already been discovered in an earlier field the detection is recorded as a rediscovery. Only the first rediscovery is recorded, for consistency with the work of D'Abramo et al. (2001). We filter potential NEOs from our survey simulator discovery output using criteria similar to those used by contemporary NEO surveys (such as thresholds on the candidate's rates of motion).

Objects may be missed by a survey for any number of reasons. (i) Objects far from Earth may not reflect enough light to exceed the limiting magnitude of the detector. (ii) Small objects may not reflect enough light to be detected, or they may pass so quickly through the survey's search volume that they streak across multiple pixels of a CCD, reducing their peak-pixel signal and chance of detection. (iii) Some objects, while large, may have such low albedos that they reflect very little light. Dark C-type NEOs are much harder to detect than brighter S-type objects. (iv) The rates of motion of some NEOs can mimic the rates of uninteresting objects so that they may not be identified as NEOs. (v) The detection software or human observer may not operate with $100 \%$ efficiency. Moving objects are typically detected by their motion against a background of stars in a set of images. If an object's image is convolved with a star or galaxy in some of the images the software might not detect the moving object.

Our simulator implicitly addresses these issues by imposing upon the survey a limiting magnitude, minimum detectable rate of motion, minimum galactic latitude, and other restrictions. Our most realistic simulator was tuned to match the operating performance of an existing wide-field survey and, in this way, implicitly incorporates all the efficiency-dependent parameters relevant to that survey.

\section{Testing the NEO model $\&$ simulator}

In the next section (Section 4) we will employ our NEO model and survey simulator to study the effectiveness of various surveying strategies and place limits on the time to completion of the NEO population. But in order to make convincing arguments about prospects for achieving the Spaceguard Goal we first need to establish that our NEO model and simulator are reasonable approximations of reality. The best way to achieve this confirmation is through comparison of our simulations with the results of actual NEO surveys.

There are many surveys with which we could compare our work if we had access to their full-pointing history and efficiency characteristics. Time constraints and lack of access to the required detailed information made it difficult or impossible to compare to all the current or historical surveys and we believe that the full suite of tests is unnecessary. Since the Bottke et al. (2002) NEO model was derived from
Spacewatch observations comparisons with that survey would not be diagnostic of problems. We chose to model the Catalina Sky Survey (CSS), which provides an interesting test because, unlike all other surveys, they adopted a high ecliptic latitude observing strategy. The most successful NEO survey to date is the LINEAR program (Stokes et al., 2000) which provides a stringent test of our model because (i) as a wide-field less deep survey it is distinctly different from Spacewatch and (ii) it provides the largest NEO statistics for the comparison. The difficulty associated with comparing our results to LINEAR is that we did not have access to LINEAR's pointing history or efficiency characterization.

\subsection{Catalina sky survey comparison}

The survey simulator and the NEO model were tested in detail against nine month's surveying history of the CSS. The full pointing history of the CSS from April to December 1999 was available to us, as well as other crucial details such as the size of the field of view, limiting magnitude, and the complicated rate "cuts" used to distinguish NEOs from other background asteroids.

We have quantified the rate cuts implemented at the CSS in the following manner. If the body is "fast" ( $>0.5^{\circ} /$ day $)$ it is considered a NEO candidate. If not, for bodies at solar elongation $<90^{\circ}$ we use a rule of thumb that compares the ecliptic latitude rate $(\dot{\beta})$ to the ecliptic longitude rate $(\dot{\lambda})$. If $|\dot{\beta}|>|\dot{\lambda}| / 2$ the object is declared as a potential NEO. For objects within $15^{\circ}$ of opposition, those with $|\dot{\lambda}|<0.12^{\circ}$ /day are also kept as candidate NEOs. Finally, if all these tests are negative, we attempt to compute a Väisälä orbit assuming that the body is at perihelion. If the computation fails, or gives a small perihelion distance or too high an inclination, the object is accepted as a potential NEO. In all other cases the object is discarded as uninteresting.

In all the following survey simulations we use three different possible characterizations of a survey's efficiency with respect to magnitude:

- limiting magnitude $\left(V_{\text {limit }}\right)$

The survey is $100 \%$ efficient (at discovering moving objects) to $V_{\text {limit }}$ and $0 \%$ for fainter objects.

- $50 \%$ efficiency point $\left(V_{50 \%}\right)$

The V-magnitude at which the survey efficiency is $50 \%$.

- magnitude drop-off range ( $\left.V_{\text {dropoff }}\right)$

The detector is $100 \%$ efficient for objects with $V<V_{50 \%}$ $-V_{\text {dropoff }} / 2$ and has $0 \%$ efficiency for objects with $V>$ $V_{50 \%}+V_{\text {dropoff }} / 2$. The efficiency drops linearly from $100 \%$ to $0 \%$ in the range $\left[V_{50 \%}-V_{\text {dropoff }} / 2, V_{50 \%}+V_{\text {dropoff }} / 2\right)$.

Note that $V_{\text {limit }}$ could be achieved with $V_{50 \%}=V_{\text {limit }}$ and $V_{\text {dropoff }}=0$ so that the two parameters are not orthogonal but are in fact mutually exclusive. We introduce this nota- 
Table 1

Model simulation comparison with CSS

\begin{tabular}{lrr}
\hline Category & \multicolumn{1}{c}{ Model } & CSS \\
\hline Total NEO detections & $30.9 \pm 6.1$ & 38 \\
Atens & $2.2 \pm 2.0$ & 2 \\
Apollos & $18.4 \pm 4.2$ & 21 \\
Amors & $10.3 \pm 2.4$ & 15 \\
\hline
\end{tabular}

tion only to convey the idea that $V_{\text {limit }}$ represents a hard limit whereas $V_{50 \%}$ is a somewhat "soft" condition on the limiting magnitude.

For the nine month period in 1999 we ran the CSS survey simulator with $V_{50 \%}=19.0$ and $V_{\text {dropoff }}=1.0$. The simulation used the exact pointing history of the telescope and its proper field-of-view. Unlike most of the other simulations presented here which consider NEOs with $H \leq 18$, this test was performed on NEOs with $H<18.3$.

To assess the rejection proficiency of the CSS implementation we ran the survey simulation on a population of 10,000 Main Belt asteroids and 1500 Hungarias. The orbital elements $(a, e, i)$ were chosen as those of known bright asteroids and the angular elements were picked randomly. We used a reasonable differential $H$-distribution with $n(H)$ $\alpha 10^{0.4 H}$. The simulated CSS detected 170 main belt asteroids and only 3 (a reasonable 98\% rejection factor) were heralded as potential NEOs. Similarly, of the 86 Hungarias detected in the simulation 11 were identified as potential NEOs, yielding a rejection factor of $87 \%$. One of us (Spahr) was a member of the CSS and believes that these are appropriate rejection factors.

Using the 15 NEO models described in Section 2.1, the simulation of the CSS produced the results shown in Table 1 . We believe the correspondence between the simulation and the CSS is good.

One of the concerns in using the model of Bottke et al. (2002) is their normalization procedure for the number of NEOs with $H \leq 18$ which relied on estimating the actual number of NEOs with $H<15$. The technique employed by Stuart (2001) did not require such an ad hoc normalization. The shape of Stuart (2001)'s one-dimensional semimajor axis and eccentricity distributions both appear to agree with Bottke et al. (2002), while their inclination distribution is more "flat." But their predicted total number of NEOs with $H \leq 18$ is about $20 \%$ higher than predicted by Bottke et al. (2002). The fact that our simulation of the CSS provides absolute numbers in good agreement with CSS observations suggests that the normalization of the Bottke et al. (2002) model is reasonable, but we note that the agreement would likely be better with the slightly higher normalization of Stuart (2001). Since the simulation and the CSS data agree, it appears that all these effects are small and that these tests certify the fidelity of the model and the survey simulator. Furthermore, since most of this work presents the relative completeness for NEOs during a simulated survey, the overall normalization is not very important-only the orbital, distributions in $(a, e, i, H)$, including the relative proportion of Aten, Amor, and Apollo asteroids, is relevant to this study.

\subsection{LINEAR survey comparison}

For the eventual purpose of determining whether a LINEAR-like survey can meet the Spaceguard Goal, for comparison to LINEAR (Stokes et al., 2000), and to add a veneer of realism to our simulations, we model a NEO search program where the entire sky is covered twice in 14 days, centered on the new moon within a lunation. The survey technique was motivated by our examination of LINEAR's monthly sky coverage as shown on the LONEOS website (http://asteroid.lowell.edu/cgi-bin/koehn/ coverage). The sky is surveyed \pm 6 hs of right ascension from opposition, beginning at southerly declinations $\left(-30^{\circ}\right)$ and moving progressively northward (to $+80^{\circ}$ ) each night. There is no overlap in the covered sections of sky from night to night and there is no consideration of the limitations imposed by the position and phase of the moon. So this idealized simulation sets an upper limit on the capabilities of LINEAR-like surveys of the sky.

We determined $V_{50 \%}$ and $V_{\text {dropoff }}$ for LINEAR using the magnitudes of the first 524 NEOs reported by them to the MPC. The values were histogrammed in bins 0.5 magnitudes wide and bins in the range $14.0 \leq V<16.5$ were fit to a function of the form $10^{a V+b}$, where $a$ and $b$ were parameters of the fit. Dividing the extrapolated function by the actual reported histogram shows that their detection efficiency begins to drop near $V=18.3$ and reaches zero near $V=19.8$ (therefore $V_{\text {dropoff }}=1.5$ ). The efficiency drops roughly linearly (no pun intended) in this range so that $V_{50 \%} \sim 19.05$. We used $V_{50 \%}=19.0$ for LINEAR in the following simulations.

In lieu of proprietary information from LINEAR (e.g., detailed pointing history for their detector and in-depth knowledge of their detection efficiency), which would have allowed us to model their survey exactly, we compare our pseudo-LINEAR simulations to results tabulated by D'Abramo et al. (2001). In their Table 1, for NEOs with $H$ $<18$, they list 273 NEOs known prior to 1 Jan 1999 (we refer to these as "pre-discovered" NEOs-those objects which were known to exist before the study took place) and 176 discovered by LINEAR from that date through 31 December 2000. Our pseudo-LINEAR simulation discovered 273 NEOs at day $620 \pm 50$. During the next $780 \pm 110$ days the simulation "discovered" 176 new NEOs-less than one-half $\sigma$ different from LINEAR's catch of the same number of objects in two years (730 days).

Figure 1 compares our pseudo-LINEAR survey's results as a function of $H$ with the actual LINEAR data as tabulated by D'Abramo et al. (2001). The topmost figure shows the $H$-distribution of the first 273 NEOs discovered by the pseudo-LINEAR simulation. The actual set of prediscovered asteroids (dashed line) is skewed to lower $H$ (brighter 

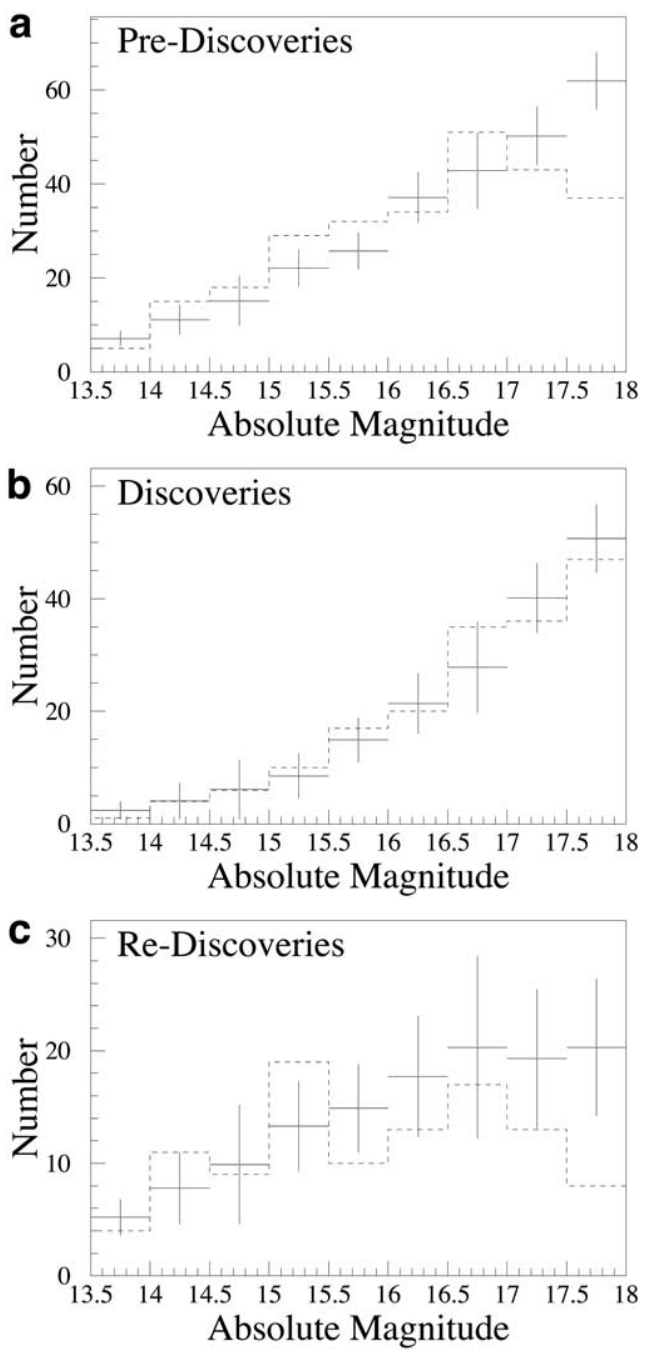

Fig. 1. The dashed histogram in each figure provides the tabulated results for the LINEAR survey by D'Abramo et al. (2001). The points with $(1 \sigma)$ error bars are the results of our pseudo-LINEAR survey with $V_{\text {limit }}=19.0$. (A) $H$-distribution of the 273 known NEOs prior to 1 January 1999 and the first 273 objects discovered with our simulation. (B) $H$-distribution of the next 176 NEOs discovered by LINEAR (1 January 1999 to 31 December 2000 inclusive) and our simulation. (C) $H$-distribution of previously known NEOs rediscovered by LINEAR and our simulation during the same time frame.

objects) than our pseudo-LINEAR simulation because the magnitude limits were considerably lower for surveys or serendipitous discoveries of NEOs prior to "modern" CCD surveys. Our pseudo-LINEAR- simulation is better at finding fainter NEOs with $H \sim 18$.

The correspondence between our simulation and the actual LINEAR data (dashed line) for the next 176 NEO discoveries is remarkably good, as shown in Fig. 1B. The fact that we are able to accurately model both the discovery rate and the $H$-distribution of the LINEAR survey implies that our simulation is a good proxy for the real survey.

Finally, during the time that the pseudo-LINEAR survey discovered the set of 176 objects it was also rediscovering the 273 objects which had been previously discovered.
Figure 1C shows the rediscovery comparison between our simulation and LINEAR. Once again, the simulation is good with a slight overabundance of rediscovered faint NEOs as a consequence of the simulation's ability to prediscover fainter objects than were actually known by 1 January 1999 .

In order to reconcile residual differences between the simulation and LINEAR as shown in Fig. 1 keep in mind that the pseudo-LINEAR simulation is not a LINEAR simulation. LINEAR overlaps sky near opposition and they do not follow the search pattern adopted in our simulation. The orbit distribution of objects which were prediscovered before LINEAR is not the same orbit distribution (because it is the sum of the distributions from many different surveys over many decades) that our simulation "prediscovered" after the first $\sim 620$ days.

\section{The search for undiscovered NEOs}

In this section we introduce the results of our modelling of various idealized and somewhat realistic surveys of the sky for NEOs. Since the objective of this work is to determine if it is possible for NEO surveys to achieve the Spaceguard Goal we present the results in figures which provide the NEO completion level as a function of calendar year. These figures are very similar to those provided by other studies except that the previous results were presented as a function of time where the starting condition represented no known NEOs. We believe that it is not usually possible to
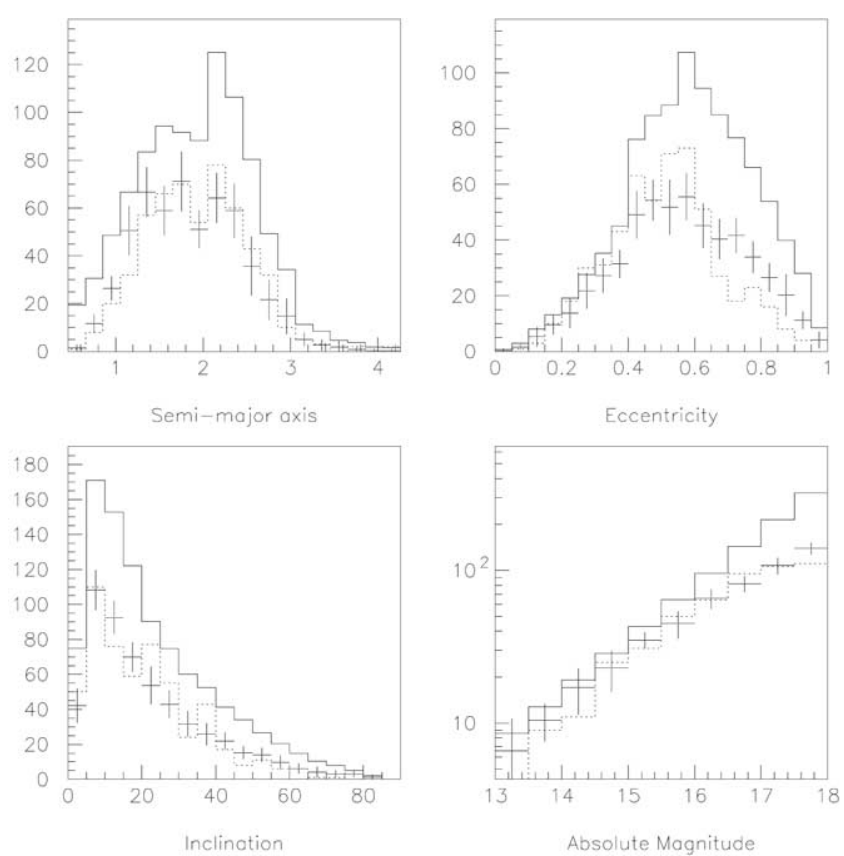

Fig. 2. The solid histograms represent the predictions of our NEO model. The dotted histograms are the known distributions of NEO orbit elements and absolute magnitudes by all search programs as of 1 January 2002 to $H$ $=18$. The points with $(1 \sigma)$ error bars are the expected distribution of the elements and $H$ using the pseudo-LINEAR simulation with $V_{50 \%}=19.0$. 


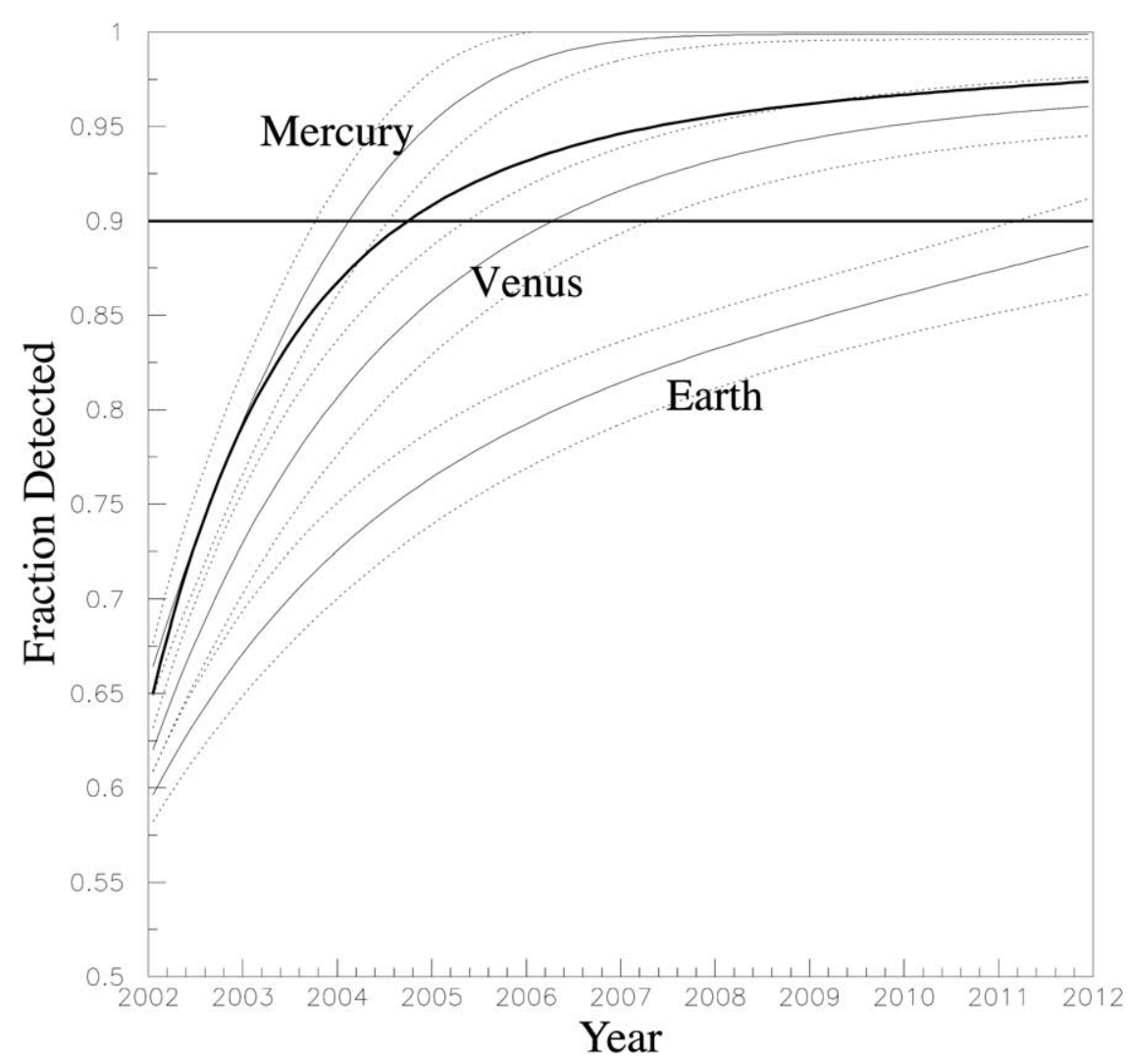

Fig. 3. Discovery completeness for NEOs with $H \leq 18$ as a function of time for space-based surveys with $V_{\text {limit }}=19.0$. The survey takes place in a circular orbit at the semimajor axis of each of the three innermost planets and covers the entire sky each day to within $45^{\circ}$ of the Sun. The dotted lines enveloping the thin solid curves show the RMS error in the fraction detected as a function of time. The thick solid curve repeats the result for the All-Sky-survey to $V_{\text {limit }}$ $=21.5$ from Fig. 4 for comparison. The solid horizontal line is at $90 \%$ completion.

determine the length of time to a given NEO completion from figures of the latter form.

The problem in determining time to completion is that $\sim 50 \%$ of all NEOs with $H \leq 18$ are already known and these NEOs have been discovered by a variety of surveys with different limiting magnitudes and efficiency characteristics. NEO surveys beginning now or in the future have to deal with the fact that the "easy" NEOs have already been discovered by previous surveys with bright limiting magnitudes and a morass of observational selection effects. The as-yet undiscovered NEOs are simply more difficult to discover. It is invalid to compare the current situation to a simulation of a NEO survey to any limiting magnitude which starts with no previously known NEOs.

Our modelling technique produces an estimate of the $(a$, $e, i, H)$ distribution for the set of currently undiscovered NEOs upon which we then run our simulations. For the purpose of readability each of our figures presenting the fraction detected (percentage completion) vs calendar year (Figs. 3-6) shows fits to the mean and RMS results rather than raw data. We were not able to fit the data to a simple exponential $(F(t)=1-\exp -t / \tau$, Harris, 1998), which assumes that all objects have the same detection probability. This is consistent with the discussion in the preceding paragraph in which we argued that the detection probability decreases as the completion level increases. In each of the figures curves of the form

$$
F(t)=F_{\max }-N \exp ^{a t+b t^{2}+c t^{3}}
$$

were used, where the quantities $F_{\max }, N, a, b$, and $c$ are all free parameters. The higher order terms provide a means of fitting the nonexponential behavior corresponding to the changing detectability of the NEOs as a function of time.

Using our model of the undiscovered NEO population we follow the lead of earlier work on this subject and simulate progressively more realistic surveys. We begin with a simulation of the ultimate NEO surveyor (a space-based platform) and proceed through more restricted penultimate approximations of reality (Earthbased all-sky and restricted surveys) and finally to a simulation which has already been shown to be somewhat realistic (pseudo-LINEAR surveys). Much of the discussion of the results of these surveys is left to Section 5.2 .

\subsection{The discovered NEO population}

Figure 2 compares the distribution of the 544 known $\mathrm{NEO}(a, e, i, H)$ values (dotted line) as of 1 January 2002 to $H \leq 18$ with the distribution for the first 544 objects found 


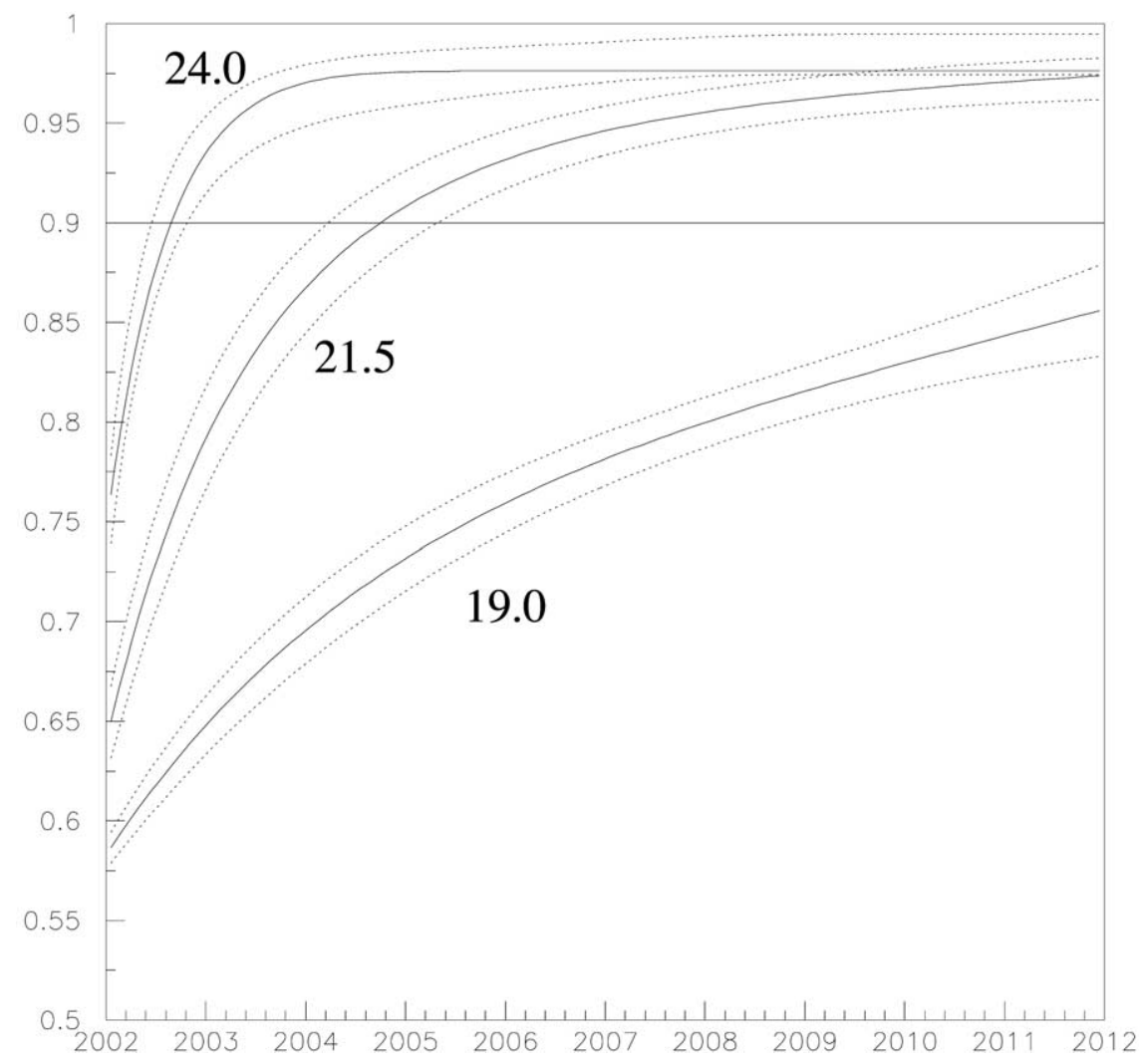

Fig. 4. Discovery completeness for NEOs with $H \leq 18$ as a function of time for $100 \%$ efficient all-sky surveys to limiting magnitudes of $19.0,21.5$, and 24.0. The dotted lines enveloping the solid curves show the RMS error in the fraction detected as a function of time. The surveys take place at Earth's equator and cover the entire dark sky every night to the specified limiting magnitude above 2.0 air masses when the sun is more than $18^{\circ}$ below the horizon. The solid horizontal line is at $90 \%$ completion.

by our pseudo-LINEAR simulation (Section 3.2). Visual comparison of the known and simulated distributions might suggest a good agreement between the two but a $\chi^{2}$ comparison shows that only the simulated $H$-distribution has a high probability of matching the observed objects. This is not unexpected as we will discuss below.

The known NEOs in Fig. 2 were discovered by more than 40 observatories over a period of more than 100 years. This makes it difficult to quantify the observational selection effects in the entire sample of known NEOs. Thus, we were surprised to find that the pseudo-LINEAR simulation could roughly reproduce the observed distribution of all known NEOs.

Of course, the agreement between the simulation and the known objects is not perfect. In general, there is a "skew" to the distribution of eccentricities-the known NEOs have a lower mean eccentricity than those identified by pseudoLINEAR. This is due to the brighter limiting magnitude for earlier or other surveys which discovered the NEOs not identified by LINEAR. Those surveys detected NEOs closer to Earth and NEOs which spend a longer fraction of time in Earth's vicinity, e.g., have lower eccentricity. More distant, higher eccentricity NEOs spend a larger fraction of their time at great distances (where they are faint) and less time in Earth's environs. Relative to our pseudo-LINEAR simu- lation this would skew the eccentricity distribution toward lower values as seen in Fig. 2.

\subsection{Undiscovered NEO population}

In the previous section we showed that our model and survey simulator are able to reproduce the gross characteristics of the one-dimensional $(a, e, i, H)$ distributions of the 544 known NEOs as of 1 January 2002. It follows that the set of 417 undiscovered NEOs obtained by running each of our 15 independent NEO models through our survey simulator until 544 NEOs are discovered are models of the set of currently unknown NEOs with $H \leq 18$.

The set of discovered NEOs is quite different from the set of undiscovered NEOs as of 1 January 2002. The mean semimajor axes for the two sets are $1.86 \pm 0.01 \mathrm{AU}$ and $2.11 \pm 0.01 \mathrm{AU}$, respectively. Similarly, the mean eccentricities and inclinations change from $0.558 \pm 0.002$ to $0.614 \pm 0.002$ and from $21.7^{\circ} \pm 0.2^{\circ}$ to $24.9^{\circ} \pm 0.2^{\circ}$, respectively. In each case the quoted error is the standard error on the mean even though the distributions are not gaussian. For each $(a, e, i, H)$ the undiscovered set is much more difficult to detect-more distant, higher eccentricity, and more highly inclined orbits will take longer to discover than the currently known set. It is for this reason that it is 


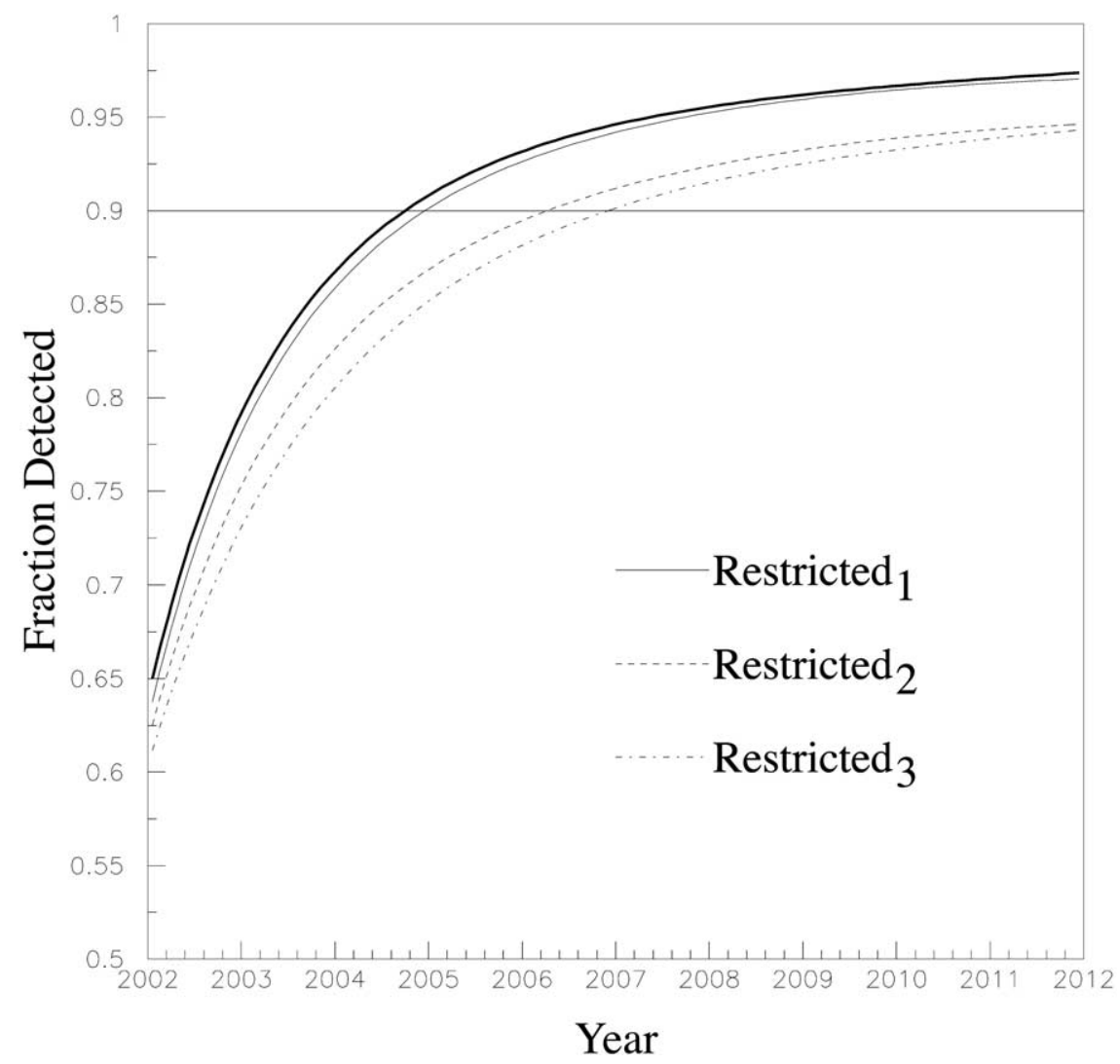

Fig. 5. Discovery completeness for NEOs with $H \leq 18$ as a function of time for restricted surveys to $V_{\text {limit }}=21.5$. The thick solid curve repeats the discovery

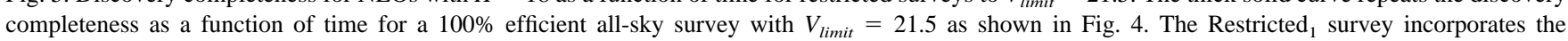
cumulative effects of excluding regions within $10^{\circ}$ of the galactic equator, observing only when the moon is less than 50\% illuminated, and observing no closer to the moon than $45^{\circ}$. Restricted ${ }_{2}$ also includes the effect of moving the observatory to a northern latitude of $35^{\circ}$ while Restricted $_{3}$ implements the requirement of accepting only objects moving faster than $0.3 \%$ day as NEO candidates. The solid horizontal line is at $90 \%$ completion. The RMS errors on the completion fraction are not shown for the sake of clarity. They are on the same order as the RMS errors in Fig. 4.

incorrect to make linear extrapolations of current discovery rates when estimating the time to a given completion level for the NEO population. The only way to estimate the time to completion is through a simulation as presented here.

Most importantly, the mean of the absolute magnitude distribution for the set of undiscovered asteroids is $17.1 \pm$ 0.8 versus $16.6 \pm 1.1$ for the discovered ensemble. In this case the reported error is simply the RMS of each distribution. So the average undiscovered NEO is half a magnitude fainter than the average known NEO and will be concomitantly more difficult to discover.

The actual set of undiscovered minor planets in the NEO population will be even more skewed toward higher eccentricities and fainter asteroids than our model suggests (see Section 3.2 and Fig. 2). Our survey simulator was not able to model well the observed eccentricity distribution and discovered too many faint asteroids relative to the known sample. So our set of 15 undiscovered NEO models are somewhat optimistic in terms of the actual undiscovered population. This would have the effect of making our estimates of time to completion for the NEO population somewhat faster than reality.

To any event, we utilize the set of 15 undiscovered NEO files in each of our survey simulations in the following sections. We are thus able to estimate the year in which NEO surveys will reach a given completeness level rather than a total number of years from the beginning of surveying as was done in all previous NEO survey simulations.

\subsection{Space surveys}

There are good motivations for locating the observing platform for NEOs in space and this option is under consideration for some proposed or planned satellites (e.g., BepiColombo, GAIA). A satellite survey system on a heliocentric orbit interior to Earth will detect IEOs and dangerous Aten class NEOs (with $a<1.0 \mathrm{AU}$ and aphelion $Q$ $\geq 0.983 \mathrm{AU}$ ), which are very difficult to detect from Earth (there are no known IEOs, yet there is every reason to believe that they exist). Tedesco et al. (2000) proposed that a space-based infrared detection system might be launched in order to supplement Earth-based NEO survey efforts. They specifically examined the efficacy of an Earth-orbiting IR surveying satellite and claim that their "strawman" satellite system would be economically competitive with a groundbased system with a limiting magnitude of 21 . In 


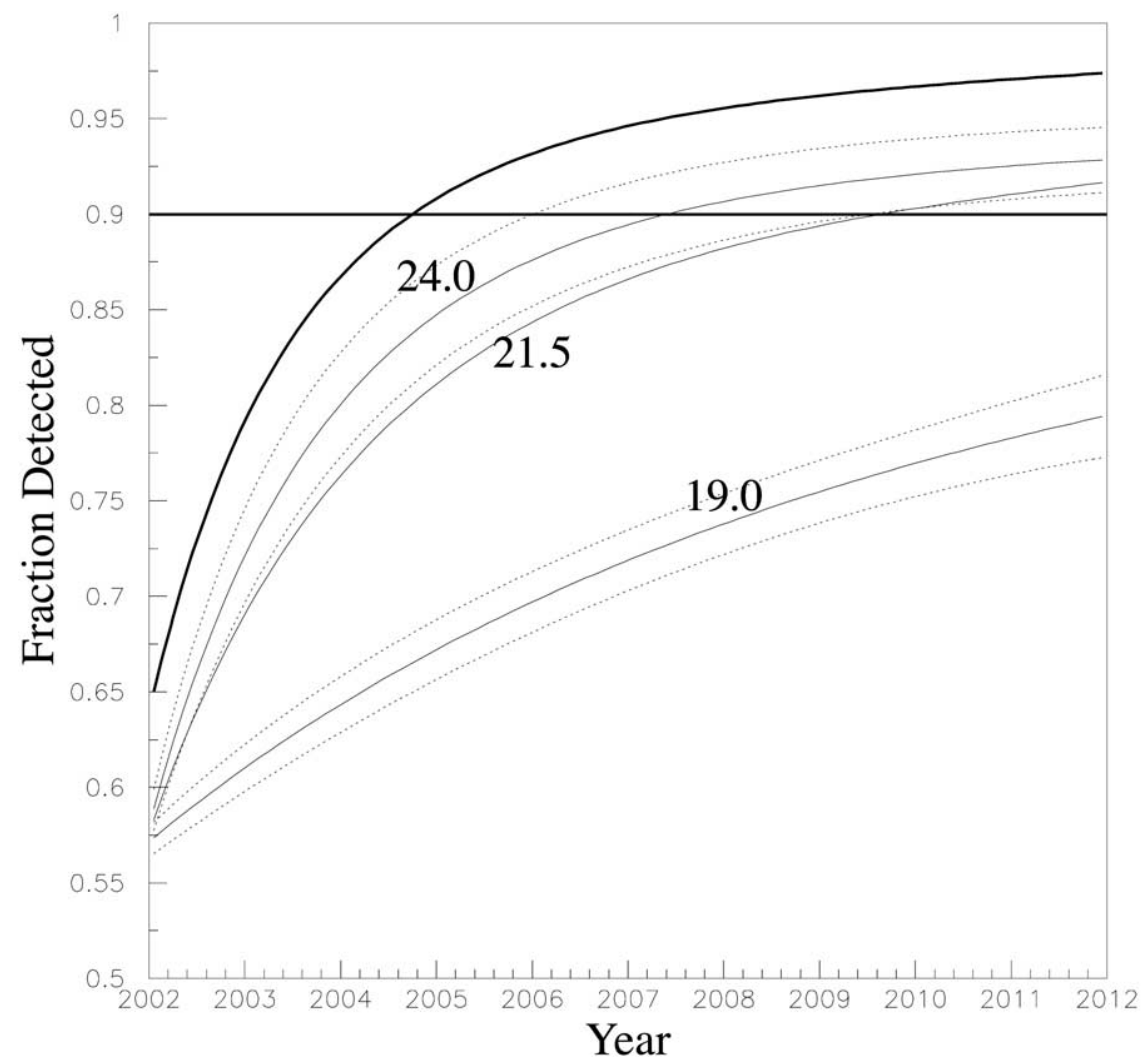

Fig. 6. Discovery completeness for NEOs with $H \leq 18$ as a function of time for pseudo-LINEAR surveys with $V_{50 \%}$ of $19.0,21.5$, and 24.0 (bottom to top). The dotted lines enveloping the $V_{50 \%}=19.0$ and 24.0 curves show the RMS error in the fraction detected as a function of time. The error on the completion fraction for the $V_{50 \%}=21.5$ line are not shown for the sake of clarity. They are on the same order as the errors on the other two lines. The thick solid curve repeats the result for the All-Sky survey to $V_{\text {limit }}=21.5$ from Fig. 4 for comparison. The solid horizontal line is at $90 \%$ completion.

keeping with the goals of this work, and unlike the detailed study of Tedesco et al. (2000), we model the performance of a "perfect" satellite surveying system to set a benchmark for this type of surveying and to allow comparison with our perfect Earth-based simulations.

We model a benchmark satellite NEO surveying system (in $V$ ) restricted only by

- minimum angular separation from the $\operatorname{Sun}\left(\theta_{\text {Sun }}=\right.$ $\left.45^{\circ}\right)$

Satellite-based telescopes (other than those devoted to solar studies) typically avoid regions of the sky located too close to the Sun.

We used $V_{\text {limit }}=19.0$ for all the space-based simulations and assumed that the entire sky outside of the cone around the Sun would be searched by the system every day. The results for surveys from Earth-, Venus-, and Mercury-like orbits are shown in Fig. 3 (the thick solid curve corresponds prematurely to an All-Sky survey to $V_{\text {limit }}=21.5$ discussed in Section 4.4 in order to facilitate comparison between the various survey types).

Note the impressive improvement in the discovery rate as the satellite's semi-major axis is reduced. This enhances the discovery rate of all NEOs but especially the Potentially Hazardous Objects (PHOs), Atens and IEOs. There are a many reasons why observing from interior to Earth's orbit is a good idea. Roughly $68 \%$ of NEOs in the Bottke et al. (2002) model have perihelia inside Earth's orbit but only about $40 \%$ and $18 \%$ have perihelia inside Venus's and Mercury's orbits, respectively. Moving the observatory closer to the Sun therefore dramatically increases the fraction of objects which may be discovered over their entire orbit. Furthermore, the NEOs appear more often at small phase angles and closer to the Sun and both effects work towards making the objects brighter as viewed from the satellite.

The advantage of a space-based over an Earth-based survey obviously depends on many factors including the relative sky-area coverage and the limiting magnitude. For instance, the time to design, construct, test, launch and operate a satellite might be so long that an Earth-based observatory could find more NEOs in the same amount of time. The decision on whether to pursue construction of a space-based platform should rest on a detailed simulation of the satellite's performance in comparison to existing and funded Earth-based systems.

\subsection{All-sky surveys}

In these simulations the entire sky visible from one location may be searched on every night. To maximize the 
Table 2

Percentage completion by 1 January 2008

\begin{tabular}{lllc}
\hline Survey type & $V_{\text {limit }}=19.0$ & $V_{\text {limit }}=21.5$ & $V_{\text {limit }}=24.0$ \\
\hline All-Sky & $80 \pm 1$ & $96 \pm 1$ & $98 \pm 1$ \\
Restricted $_{1}$ & $79 \pm 1$ & $95 \pm 1$ & $98 \pm 1$ \\
Restricted $_{2}$ & $76 \pm 1$ & $92 \pm 1$ & $96 \pm 1$ \\
Restricted $_{3}$ & $76 \pm 1$ & $91 \pm 1$ & $94 \pm 1$ \\
Pseudo-LINEAR $_{\text {Satellite (Earth) }}$ & $74 \pm 2$ & $88 \pm 2$ & $91 \pm 2$ \\
Satellite (Venus) & $93 \pm 2$ & $97.7 \pm 0.9$ & $99.7_{-0.4}^{+0.3}$ \\
Satellite (Mercury) & $99.7_{-0.6}^{+0.3}$ & $99.9_{-0.3}^{+0.1}$ & $100.0_{-0.2}^{+0.0}$ \\
\hline
\end{tabular}

searchable area of sky the All-Sky surveys take place from an observatory located on Earth's equator. They allow us to set an upper limit on the ability of any Earth-bound survey and a benchmark for "optimizing" surveys. In our opinion, an Earth-based survey is "optimized" when it is performing as well as possible compared to an all-sky, every-night, $100 \%$ efficient survey. It is always possible to imagine a better survey by increasing the size of the telescope, computing power, CCD efficiency, etc.

The all-sky surveys do not explicitly take into account that each survey location must be visible for a time period long enough to establish motion of NEO candidates. This could easily be mimicked by artificially increasing the maximum allowable air mass at which surveying can take place. In other words, for the current simulation, a single observation of the object is considered a "detection." Otherwise, the limited list of restrictions on these surveys is as follows:

- minimum solar zenith angle $\left(z_{\min }=108^{\circ}\right)$

The minimum allowable angle between the center of the Sun and the zenith. We always use the standard definition for the end of astronomical twilight which is equivalent to requiring that it be dark while performing a survey.

- maximum air mass $\left(Z_{\max }=2.0\right)$

Astronomical observatories typically avoid scanning too close to the horizon where atmosphere-induced extinction and bad seeing reduce the limiting magnitude of the survey.

We chose three limiting magnitudes as our canonical reference points which correspond roughly to those for the LINEAR survey $\left(V_{\text {limit }}=19.0\right.$ - see Section 3.2), Spacewatch survey $\left(V_{\text {limit }}=21.5\right.$ - Jeffrey Larsen, personal communication, 2001), and the proposed limit $\left(V_{\text {limit }}=24.0\right)$ for the ambitious Large Synoptic Survey Telescope (LSST).

The results for 10-year surveys to $V_{\text {limit }}=19.0,21.5$, 24.0 are shown in Fig. 4. It is clear that no NEO survey to $V_{\text {limit }}=19.0$ can achieve $90 \%$ completeness by 2008 to meet NASA's commitment. Table II shows that by 2008 an All-Sky survey to this limiting magnitude would achieve 80 $\pm 1 \%$ completion. On the other hand, Table III shows that it would require $15 \pm 2$ years (from 1 January 2002) to reach $90 \%$ completeness. The time to $90 \%$ completion for the $V_{\text {limit }}=21.5$ and 24.0 cases are dramatically reduced
(2.8 \pm 0.3 and $0.6 \pm 0.1$ years respectively) and by 2008 they reach $96 \pm 1 \%$ and $98 \pm 1 \%$ completeness respectively. These survey's fainter limiting magnitudes allow them to seek NEOs at greater heliocentric and geocentric distances thereby increasing the volume of space accessible for discovery. Alternatively, given our NEO model, it would require an All-Sky survey to $V_{\text {limit }}=20.1 \pm 0.2$ in order to achieve $90 \%$ completion by 2008 .

For comparison, Bowell and Muinonen (1994) (Fig. 4) whole-sky survey simulations to $V_{\text {limit }}$ of 22 and 24 achieve $90 \%$ completeness after $\sim 1.8$ and 0.2 years respectively. Their simulations studied discoveries of NEOs $\geq 1.0 \mathrm{~km}$ diameter. While our All-Sky surveys may be unrealistically ambitious, their whole-sky surveys require searching the sun-lit sky as well! The most direct comparison, for the $V_{\text {limit }}=24$ systems, indicates that our estimated time to completion is about $5 \times$ longer than their calculation. The tremendous difference is most likely due to their searching during the day but there is also undoubtedly a contribution from the differences in our orbit distributions. Discovery of NEOs in the direction near the Sun is advantageous (it is one of the reasons that space-based NEO surveys may outperform Earth-based surveys) as the density of NEOs above a given magnitude threshold tends to increase at small solar elongations (as it does near opposition).

\subsection{Restricted surveys}

The super-surveys discussed in the previous section are clearly unrealistic, though they are illustrative of the best performance achievable under minimal restrictions. A more realistic survey simulation (yet still limited only by physical rather than funding, technological or computational considerations) involves the following:

$$
\text { - } \text { minimum galactic latitude }\left(b_{\text {min }}=10^{\circ}\right)
$$

Most NEO surveys avoid the region near the galactic equator. The high star density confuses the automated software (and tires the human surveyor) and increases the false detection rate of all objects dramatically. The LINEAR group (Stokes et al., 2000) does survey through the Milky Way but they have reduced NEO detection efficiency within about $20^{\circ}$ of the galactic plane.

Table 3

Surveying years required to achieve $90 \%$ completion

\begin{tabular}{lccc}
\hline Survey type & $V_{\text {limit }}=19.0$ & $V_{\text {limit }}=21.5$ & $V_{\text {limit }}=24.0$ \\
\hline All-Sky Restricted $_{1}$ & $15 \pm 2$ & $2.8 \pm 0.3$ & $0.6 \pm 0.1$ \\
Restricted $_{2}$ & $17 \pm 2$ & $3.0 \pm 0.4$ & $0.7 \pm 0.1$ \\
Restricted $_{3}$ & $22 \pm 3$ & $4.2 \pm 0.5$ & $1.3 \pm 0.3$ \\
Pseudo-LINEAR & $33 \pm 5$ & $4.8 \pm 0.6$ & $3.0 \pm 0.4$ \\
Satellite (Earth) & $12 \pm 2$ & $8 \pm 1$ & $6 \pm 1$ \\
Satellite (Venus) & $4.2 \pm 0.6$ & $1.4 \pm 0.3$ & $0.4 \pm 0.1$ \\
Satellite (Mercury) & $2.1 \pm 0.2$ & $1.2 \pm 0.2$ & $0.030 \pm 0.007$ \\
\hline
\end{tabular}


- minimum angular separation from the moon $\left(\theta_{\text {moon }}=\right.$ $\left.45^{\circ}\right)$

Surveying near the moon typically induces light scattering within the dome and telescope optics. This produces a variety of effects on the image which confuse detection software.

- maximum moon illumination $\left(f_{\max }=50 \%\right)$

All existing and prior NEO surveys search during "darktime" centered on the new moon. In these simulations surveying takes place only on nights when the moon is illuminated by less than this value.

- observatory latitude $\left(\phi=35^{\circ}\right)$

The All-Sky surveys take place on the equator $\left(\phi=0^{\circ}\right)$ to maximize sky coverage, but most contemporary NEO surveys are located in the northern hemisphere and at nonzero latitudes.

- minimum discoverable rate of motion $\left(\omega_{\min }=\right.$ $0.3 \%$ day)

Distant NEOs may be moving fast enough to be detected yet too slowly to be identified (discovered) as NEO candidates. NEO surveys which limit their searches to regions near opposition enjoy a luxury in that apparent asteroid rates of motion are well correlated with orbital elements opposite the Sun. Main Belt asteroids, typically with small eccentricities, have rates of motion confined to a narrow range around $(\dot{\lambda} \sim-0.25 \%$ day, $\dot{\beta} \sim 0.0 \%$ day $)$. More distant asteroids (trojans, centaurs, Kuiper belt) have much slower rates in ecliptic longitude while NEOs, with relatively small $a$, high $e$, and high $i$, can appear with wide-ranging ecliptic longitude and latitude rates. A threshold $\omega_{\min }$ on the total rate of motion $\omega=\sqrt{(\dot{\lambda} \cos \beta)^{2}+(\dot{\beta})^{2}}$, or ranges in $(\dot{\lambda}, \dot{\beta})$ for potential NEO identification, is usually sufficient for these opposition surveys. Wider ranging NEO surveys would benefit by employing ecliptic position-dependent ranges in $(\dot{\lambda}, \dot{\beta})$ for superior NEO identification. We note that surveys which report all asteroid detections eventually identify NEOs after linking many nights of observations through an orbit determination. We used $\omega_{\min }=0.3^{\circ}$ day because it is about the maximum rate of motion of main belt asteroids at opposition and because it was the rate limit for reporting unusual asteroids to the MPC by LINEAR.

Figure 5 shows the effect of imposing these restrictions in parallel on the All-Sky survey with $V_{\text {limit }}=21.5$. (Tables 2 and 3 summarize the results in a consistent manner for all three limiting magnitudes.)

The thick solid line in the figure reproduces the mean results for the All-Sky survey $\left(V_{\text {limit }}=21.5\right)$. The Restricted $_{1}$ curves take into account the effects of scanning restricted to $b>10^{\circ}, \theta_{\text {moon }}>45^{\circ}$, and $f_{\max }=50 \%$ and almost overlap the All-Sky curve. These restrictions have little effect on the otherwise pervasive coverage of the survey. The moon changes its phase, moves out of the way, and exposes asteroids which were previously hidden. Asteroids eventually move out of the galactic plane and are discovered.

On the other hand, the next curve $\left(\right.$ Restricted $\left._{2}\right)$ includes the effect of scanning at a nonequatorial latitude $\left(\phi=35^{\circ}\right)$. The northerly latitude and airmass constraint limit the lowest ecliptic latitude at which objects can be discovered, thereby eliminating the possibility of finding high-inclination objects currently in the southern hemisphere. The length of time to $90 \%$ completion increases by $1.4 \pm 0.6 \mathrm{yrs}$ with that level of completion being achieved in $2006.2 \pm$ 0.5 . It is important to note that the equatorial survey finds more objects than the higher latitude survey at all inclinations.

The Restricted ${ }_{3}$ curve in Fig. 5 show the effect of adding a minimum rate of motion requirement in order for the NEO to be discovered as such by the surveying system. This restriction is not as tight as that imposed by surveying at a nonequatorial latitude because every asteroid has some opportunity during the time it is in the night sky to display a rate of motion greater than the specified minimum. As suggested above, the reduction in the completeness due to this cut could easily be reduced by utilizing a more reasonable, position-dependent, rate cut on the detection system.

\subsection{Pseudo-LINEAR surveys}

In Section 3.2 we established that our pseudo-LINEAR survey simulator did a good job of mimicking the performance of the actual LINEAR system (Stokes et al., 2000) as tabulated by D'Abramo et al. (2001) for the years 1999 and 2000. Thus, we consider this simulator to be a realistic measure of the performance of NEO surveys compared to the idealized systems described above. The twice monthly scanning pattern to $V_{50 \%}=24$ is reminiscent of the area coverage and magnitude depth anticipated for the LSST, so these results could be viewed as a superficial analysis of the performance of that system.

Figure 6 displays the results of three pseudo-LINEAR surveys to the same limiting magnitudes used in the All-Sky surveys with the scanning pattern described in Section 3.2. The area limitation causes a dramatic reduction in discovery completeness as compared to the All-Sky surveys and also has the effect of diminishing the benefit of a very deep survey.

Unfortunately, if our simulations are correct, this implies that the LINEAR detector system (as it performed in 1999 and 2000), and even a combination of similar systems dispersed around the world could not achieve the Spaceguard goal. Table 3 shows that a pseudo-LINEAR system to $V_{50 \%}$ $=19.0$ would not achieve $90 \%$ completion to $H=18$ until the year $2035 \pm 5$ !

Since LINEAR and other survey programs continue to improve their capabilities the actual time to $90 \%$ completion is most likely considerably better than 2035. Our best estimate as of mid-year 2002 for all systems currently in 
operation is that the Spaceguard Goal will be met in 2014.1 \pm 1.2. This corresponds to an effective limiting magnitude for the combined surveys of about $V=20.5$.

Table 3 indicates that the pseudo-LINEAR survey to $V=$ 24 could meet the Spaceguard Goal since it would reach 90\% completion in $2008 \pm 1$-assuming that surveying with such an impressive system began on 1 Jan 2002. The performance of the system with reduced nightly coverage is limited by asteroids not appearing above the $V=24$ limit in that portion of sky in which the survey is imaging. After 10 years of surveying the $V=21.5$ and $V=24.0$ systems reach $91.7 \pm 1.8$ and $93.0 \pm 1.7$ completion respectively-technically the same to within the errors of this analysis. So the incremental benefit of developing a $V_{50 \%}=24.0$ system compared to one with $V_{50 \%}=21.5$ decreases as the nightly sky coverage decreases and as the requirement on time to 90\% completion relaxes (Discussed in more detail in Section 5.2).

It is very important to note that our pseudo-LINEAR survey is not the actual LINEAR survey. Our simulation provides a good match to the performance of the actual system for calendar years 1999 and 2000 as described in Section 3.2, effectively anchoring and normalizing our simulations to the system's performance in that time frame. Since the LINEAR crew continuously improves its performance in both area coverage and limiting magnitude they will quickly, and most likely already have, rendered obsolete our pseudo-LINEAR simulation's similarity to their performance. However, with their existing telescopes and detectors they are unlikely to reach $V_{50 \%}=21.5$ without seriously compromising their sky area coverage.

\section{Discussion}

Most estimates of the actual NEO population (excluding LPCs) suggest that the set of currently known NEOs represents about $50 \%$ of the total. Since it is simply a matter of time until we achieve $90 \%$ completion, the utility of estimating the actual time might seem unnecessary. The problem is that simple linear extrapolations of current NEO discovery rates can not provide meaningful estimates and will mislead funding agencies, colleagues and the public into believing that the problem has been solved and that the Spaceguard Goal is achievable.

Given the success of the existing programs it is evident that the ambitious project suggested in the seminal Spaceguard Report (Morrison et al., 1992) using six 2.5-m aperture telescopes distributed around the world in both longitude and latitude would have been overkill. They predicted that the system would achieve the goal of identifying $90 \%$ of PHOs larger than a kilometer in diameter within 25 years of operation. Our studies suggest that more modest systems can actually achieve this goal and in less time.

Most of the enthusiasm for the prospect of meeting the Spaceguard Goal is due to the success of the LINEAR asteroid survey program (Stokes et al., 2000), which has singlehandedly increased the asteroid observation rate reported to the Minor Planet Center by a factor of 10 . Their prodigious NEO discovery rate prompted some astronomers to suggest that the Spaceguard goal might be achieved within the first decade of the 21 st century. However, our studies suggest that it is very unlikely that LINEAR and even all existing programs combined can meet the Spaceguard Goal by 2008 .

In the following two sections we compare this work to the results of earlier survey simulations and discuss why the results differ. We then discuss implications of this work on how to achieve $90 \%$ completion of NEOs in a reasonable time frame.

\subsection{Comparison with previous work}

In the Introduction (Section 1) we touched upon other NEO survey simulations performed in the last decade which are of relevance to the current study. We chose not to reproduce the specific efforts of those works but instead introduced our own vantage point and a few wrinkles including a new and improved orbit distribution for the NEOs and an analysis of the statistical and systematic errors associated with simulating the surveys due to natural variations in the proposed orbit distribution. In a broad sense, our simulations agree with the general conclusions of those previous studies. The time to $90 \%$ completion varies, likely due to differences in the input NEO orbit and size distributions.

The comprehensive effort of Bowell and Muinonen (1994) extended the work which formed the basis for the Spaceguard Report (Morrison, 1992). Their NEO model was that of Rabinowitz et al. (1994). One-dimensional projections of $(a, e, i, H)$ in that model and the one adopted for this work (Bottke et al., 2002) appear similar. Like this work, they began by studying the performance of a wholesky survey and determined that in this scenario 93\% completeness could be achieved for their Earth-crossing asteroids (ECA $-q<1.13 \mathrm{AU}$ ) with $D>1 \mathrm{~km}$ using a system with $V_{\text {limit }}=20$ in only 10 years. Unlike this paper, they incorporated the extra complicating step of converting from absolute magnitude into asteroid diameter, assuming that the size distribution contains equal numbers of S- and $\mathrm{C}$-class asteroids in a diameter-limited sample. The chasm between the real and idealized worlds is exposed in their simulations which take a variety of losses into account (e.g., trailing losses, confusion with main-belt asteroids, stars and galaxies, weather, etc.) and in which $V_{\text {limit }}$ must be pushed to 22 in order to achieve $\sim 90 \%$ completeness in 25 years.

Based on our pseudo-LINEAR simulations their estimate appears to be pessimistic. We were able to model the discovery and rediscovery characteristics of the LINEAR survey which implicitly incorporates the various real-world effects they accounted for explicitly. Table 3 shows that a 
survey to $V_{\text {limit }}=21.5$ would likely achieve $90 \%$ completion in about 8 yr.

Muinonen (1998) further extended the work of Bowell and Muinonen (1994) by accounting for NEO follow-up. Discovering NEOs is of little consequence if their orbits cannot be determined with sufficient accuracy for future recovery and as input to dynamical integrators to identify those objects which have some probability of striking Earth. Unfortunately, the probability of discovering an object increases with magnitude up to the system's $V_{\text {limit }}$ due to the exponential growth in the number of NEOs as $H$ increases. Since most objects will be near a system's detection limit at discovery he incorporated a follow-up requirement for NEO discoveries and studied its impact on the overall discovery rate. The results were similar to those presented in Bowell and Muinonen (1994) with a somewhat reduced (as would be expected with the extra follow-up condition) prediction of about $85 \%$ completeness for ECAs with $D$ $>1 \mathrm{~km}$ in $25 \mathrm{yr}$.

We believe this to be a pessimistic completion estimate for a system with $V_{\text {limit }}=22$ and 25 years of surveying. The next generation of NEO surveys will likely be nearly fullsky surveys in which much of the sky is covered multiple times per month. The promise of these surveys is that they will provide their own followup. Indeed, predicted discovery rates for these surveys would probably swamp all recovery efforts-they must provide their own followup! Even though they may not be able to reobserve every object during a single apparition there is a good chance that it will become visible on a subsequent apparition for followup and orbit linkage.

Muinonen (1998) utilized an ECA model which had a "distribution of ECA MOIDs ${ }^{1}$ [which] peaks sharply at 0 AU because of large numbers of ECA on low- $i$ orbits." While this is only a qualitative description of the type of orbits in his generated model it appears to be different from our distribution. In our model, the number distribution in inclination drops precipitously for $i<5^{\circ}$ and the MOID $<$ $0.04 \mathrm{AU}$ distribution shows an excess of only $13 \%$ over what would be expected by extrapolating an exponential fit in the range $0.04 \mathrm{AU}<\mathrm{MOID}<0.30 \mathrm{AU}$. The differences in the modelled NEO distributions would certainly have an impact on the length of time to completion. In this case, since our model possesses fewer low- $i$ and (likely) small-MOID objects, it would probably increase the time to completion over those determined by Muinonen (1998).

Harris (1998) introduced a clever technique for speeding up survey simulations based on the correlation between $H$ and $V$. He generated a large set of potentially hazardous asteroids (PHAs) based on the known NEOs at the time and, for each month and asteroid, saved their sky position and

\footnotetext{
${ }^{1}$ Minimum Orbital Intersection Distance: The minimum distance between the osculating orbits of two objects. If there are no protective resonances between them it also represents their minimum possible separation.
}

$\Delta m$, where $V=H+\Delta m$. Simulating a survey then consisted of specifying the sky coverage and an $H$ distribution. $\mathrm{He}$ also provided analytical fits to his results to elucidate and extrapolate survey performance. One of his major conclusions was that an optimal NEO survey strategy relaxes the requirements on limiting magnitude in order to increase sky coverage. To achieve $>90 \%$ PHA completion for objects with $D>1 \mathrm{~km}$ would require several 2- to $3-\mathrm{m}$ aperture telescopes. Once again, our results indicate that these system requirements may be unnecessarily extravagant to meet the Spaceguard Goal.

It is important to stress that we are not implying that at the time of the earlier studies the suggested system requirements were extravagant. System capabilities have improved dramatically in the past few years and what was once thought nearly impossible is now routine or at least within reach. It is for this reason that we have specifically ignored any serious attempt to link our results to system requirements.

Beyond Harris (1998)'s simulation methodology the major difference between his work and ours is the choice of the NEO orbit distribution. His is biased by the observational parameters of each of the survey systems used to detect the known sample of PHAs at the time. It is not surprising that his generated orbit distribution is dramatically different than the PHOs from the bias-corrected results of Bottke et al. (2002).

To test the effect of radically different orbital distributions on the results of a survey simulation we ran a pseudoLINEAR simulation with $V_{\text {limit }}=19.8$ on our set of PHOs. Harris (1998) performed a simulation to the same limiting magnitude, which provided a large area coverage similar to that obtained in our pseudo-LINEAR surveys. After 10 years (125 lunar months), Harris's simulated survey had a completion fraction for PHAs of 94\%-respectable and satisfying the Spaceguard Goal. Our simulation yielded only $77 \pm 1 \%$ completion in the same time and thus would not meet the Spaceguard goal by almost $15 \%$ after 10 years. The discrepancy is most likely due to the assumed orbital distribution of the PHOs and highlights the link between a NEO model and the results of a simulation which may influence funding or political decisions. The model of Bottke et al. (2002) tends to possess PHOs with much higher mean $a$ and mean $e$, and a high tail in the inclination distribution, all of which make the generated PHOs more difficult to detect than in Harris's model.

In 2000 the "Report of the UK Task Force on Near Earth Objects" (http://www.nearearthobjects.co.uk/downloads/ full_report.pdf) compiled several recommendations related to surveying and discovering NEOs. One of the primary suggestions was that the goal for finding NEOs should be stretched to discovering $90 \%$ of objects with diameters greater than $300 \mathrm{~m}$. This more ambitious goal will be addressed in the following section. Another recommendation was that a 3-m-class telescope should be built in the southern hemisphere to survey for NEOs much smaller than 
current surveys are capable. Whereas this type of facility would always be welcome, our work suggests that there may be little benefit to survey locations which are spread in longitude or latitude (discussed in more detail in Section 5.2).

Another of the UK Task Force's recommendations was to explore the option of piggybacking a NEO survey on existing plans for a satellite. In particular, they suggested the GAIA and BepiColombo missions planned by the European Space Agency (ESA) as parasitic targets for a NEO mission. GAIA is expected to orbit the L2 point of the Sun-Earth system for 5 years. BepiColombo will orbit and study Mercury, requiring a 2.5-yr cruise time and enjoying only one year in orbit. Both spacecraft offer tempting hosts for NEO surveys.

In Section 4.3 we presented results for NEO surveys from space-based systems and showed that a survey at Mercury's orbit could be a prodigious discoverer of NEOs. The difficulty in utilizing BepiColombo would be to achieve suitable limiting magnitude and sky coverage to justify the mission when compared to an Earth-based system. A survey from Mercury to $V_{\text {limit }}=19.0$ could achieve $90 \%$ completion for the NEOs in $2.1 \pm 0.2$ years! Unfortunately, this is a couple of times longer than BepiColombo's planned lifespan at Mercury's orbit and assumes all sky coverage every day. Detailed studies of achievable completeness using this satellite as an observation platform will be required in order to justify its use instead of, or in addition to, Earth-based systems.

Mignard (2001a) and Mignard (2001b) provide a detailed simulation of the GAIA satellite's ability to detect NEOs using the same 4-dimensional orbital element distribution used in this paper. He claims that GAIA documentation (GAIA, 2000) states that it "will provide a detailed census of these objects [NEOs] down to the 20th magnitude" and goes on to show that a 5-yr survey with GAIA will achieve $\sim 50 \%$ completion for NEOs with $H \leq 18$ (at least one detection of the asteroid with the satellite's sensors). However, his studies did not utilize our technique of distinguishing between the known and undiscovered NEOs. Since the known population is already $50 \%$ complete and will become even more so in the time interval until GAIA completes its five year mission it is, once again, questionable whether GAIA can make a significant contribution to achieving the Spaceguard Goal or even finding a large number of unknown NEOs. However, GAIA's NEO survey would be parasitic to its main mission, so it is likely that a dedicated NEO satellite would yield a considerably better return.

\subsection{Implications of this work}

We believe that there is tremendous utility in simulating surveying strategies for NEOs and that our results can be used to guide thinking on future systems or likely performance of any existing or upgraded programs. Here we highlight some of our findings which we think are of interest to the NEO community.

- Multi-hemispheric surveying

We examined the performance of two simultaneous $V_{\text {limit }}$ $=21.5$ Restricted $_{2}$ sky surveys (as in section 4.5) at both $\pm 35^{\circ}$ latitude. Recall that the Restricted ${ }_{2}$ surveys incorporate the effect of not scanning in the galactic plane or when the moon is too bright or too close to the moon and from a non-equatorial location. We have already shown that a survey from a high-latitude site will not perform as efficiently as a similar equatorial version. Contrary to what might be thought, the high-latitude survey finds fewer NEOs at all inclinations. Furthermore, the performance of the two mirror-image surveys in the northern and southern hemisphere $\left( \pm 35^{\circ}\right.$ latitude) working in tandem took about $10 \%$ longer to reach $90 \%$ completion than a single equatorial survey! On the other hand, the duplicate system outperformed the equatorial survey by about $1 \%$ after $10 \mathrm{yr}$.

A comparison of the duplicated north-south systems to an equatorial-only system is not realistic given the current preponderance of surveys in the northern hemisphere. It turns out that adding a southern Restricted $_{2}$ survey to a single northern Restricted ${ }_{2}$ survey shaves about $25 \%$ off the time to $90 \%$ completion. Once again, the choice of whether it is beneficial to build a southern hemisphere survey will depend on the cost and other logistical issues and should be based on simulations such as the one presented here. Duplicating an existing or building a new northern hemisphere telescope might turn out to be an even better option.

- Space-based vs Earth-based surveying

When other non-monetary conditions are equal, our idealized space-based surveys are superior to the idealized Earth-based surveys. More of the sky is available at any time and the search can take place $24 \mathrm{~h}$ a day. Furthermore, it is well known that the sky-plane density of NEOs increases in the direction towards the Sun so pushing the survey into this region from a satellite's viewing platform offers a tremendous benefit in the rate of NEO detections. If the viewing location is from an orbit interior to Earth's then the opportunity arises to discover asteroids whose orbits are mostly or even entirely interior to Earth's orbit.

The geometrical viewing circumstances are so favorable that space-based systems may still outperform Earth-based systems even with reduced limiting magnitude or sky-coverage. Figure 3 shows that a satellite in a Mercury-like orbit with $V_{\text {limit }}=19.0$ would outperform a similar Earth-based survey with $V_{\text {limit }}=21.5$. It is also likely that restricting the sky coverage from a satellite to a region in a band around the ecliptic, and doing so only over a matter of days rather than every day, would not drastically cut the discovery rate.

Tables 2 and 3, as well as Fig. 3, show that the efficiency of NEO surveying improves as the space-based survey's orbit moves closer to the Sun, as discussed in section 4.3. 
The improvement in efficiency occurs for all classes of NEOs but is particularly poignant for Atens and IEOs.

\section{- Limiting magnitude for the Spaceguard Goal}

Utilizing our pseudo-LINEAR surveys as proxies for realistic NEO searches Table 2 indicates that meeting the Spaceguard Goal would require immediate and continued operation of a system with $V_{50 \%} \sim 24$ (with $V_{\text {dropoff }}=1.5$ as in section 3.2). Assuming that surveying with such an impressive system began on 1 Jan 2002 it would reach $90 \%$ completion in $2008 \pm 1$-probably and barely meeting the Spaceguard Goal. The area limitation of the pseudo-LINEAR survey causes a dramatic reduction in discovery completeness as compared to the All-Sky surveys and diminishes the benefit of a very deep survey. After 10 years of surveying (by the end of 2012) the $V_{50 \%}=21.5$ and $V_{50 \%}=24.0$ systems reach $92 \pm 2 \%$ and $93 \pm 2 \%$ completion, respectively - the same to within the errors of this analysis. So the incremental benefit of developing a $V_{50 \%}=$ 24.0 system compared to one with $V_{50 \%}=21.5$ decreases as the nightly sky coverage decreases and as the requirement on time to $90 \%$ completion relaxes.

The deeper survey is only marginally more beneficial because it is capable of detecting NEOs at distances greater than they can achieve. At opposition the faintest asteroid in our model (with $H=18$ ) would have $V=24.0$ at a heliocentric distance of about 4.5 AU. More than $99 \%$ of the NEOs in the model have a semimajor axis less than 4.5 AU and $\sim 85 \%$ have an aphelion less than this distance. Thus, there is a dramatic difference between the distances at discovery for the $V_{50 \%}=19.0$ and 24.0 systems but only a moderate difference between the 21.5 and 24.0 simulations. If an asteroid is not within the range of the less powerful system it will also most likely not be in range of the system with fainter limiting magnitude.

The implication of our study for the prospects of the pseudo-LINEAR system (our proxy for the actual LINEAR survey or systems like it) are not good (i.e., Table 3). Singlehandedly, it will be impossible to meet the Spaceguard Goal. If the LINEAR system (as it existed in 1999-2000) continued to be the only major discoverer of NEOs it would take until 2035 \pm 5 to achieve $90 \%$ completion to $H=18$. Even if it were possible to reproduce similar systems and distribute them around the world, so that nightly coverage of the entire sky was always possible, it would take another $22 \pm 3$ years to reach the holy grail of $90 \%$ completion. Since the actual LINEAR program has shown tremendous resourcefullness in regularly improving their search capability (indeed there are already strong indications that their current performance exceeds those upon which our pseudo-LINEAR simulation was based) it is probable that the actual time to completion will be less than predicted here.

- Extending $90 \%$ completion to $H=20.5$

When we stretch the NEO requirement to surveying for asteroids of only $300 \mathrm{~m}$ diameter (below the globally cata- strophic impact size of $1 \mathrm{~km}$ ) it is necessary to push $V_{\text {limit }}$ in order to achieve $90 \%$ completion in a reasonable time frame. The "Report of the UK Task Force on Near Earth Objects" urged that NEO surveying push discovery completion to this size range $(H \sim 20.5)$. Morbidelli et al. (2002) have shown that NEOs of this diameter would deliver Earth impacts of about 1000 megatons TNT-equivalent on average every 64,000 years. The current collision probability-weighted completeness of objects with $H \leq 20.5$ is about 18\% (Morbidelli et al., 2002). At the same heliocentric and geocentric distance and phase angle, an asteroid of this diameter will be only $9 \%$ as bright as (or about 2.6 magnitudes fainter than) a $1-\mathrm{km}$ diameter asteroid of the same albedo. We argued earlier that an Earth-based pseudoLINEAR system with $V_{\text {limit }}=21.5$ might be a good compromise for detecting NEOs with $H \leq 18$. Therefore, to achieve roughly the same results for $H \leq 20.5$ asteroids would require a limiting magnitude of $\sim 24$.

There are a limited number of solutions to the problem of meeting the Spaceguard Goal: (1) the limiting magnitude of the NEO surveying systems needs to be pushed to fainter limiting magnitude; (2) the surveying needs to be moved into space, preferably from a platform interior to Earth's orbit; (3) admit that the Spaceguard goal will not be achieved and wait a longer period of time to achieve the desired level of completeness.

\section{Conclusion}

Using a new model of the orbit and absolute magnitude distribution for NEOs and realistic simulations of asteroid surveys, we have shown that the Spaceguard goal cannot be achieved by existing systems (as of 1999-2000). There are some proposed or funded systems which might achieve $90 \%$ completion for $H \leq 18$ NEOs, but whether they can be built and do so by 2008 is questionable.

Linear extrapolations of current discovery rates into the future ignore the fact that as we discover more NEOs the ones left undiscovered are more difficult to find. Previous studies of this kind often used biased NEO orbit distributions which should have predicted more optimistic estimates for the time to meet the Spaceguard goal. It is almost paradoxical that most of those studies concluded that more powerful survey systems would be required to achieve the Spaceguard Goal than we suggest. Thus, the use of realistic NEO orbit models will be key in deciding which direction to pursue in order to eliminate the NEO hazard.

An Earth-based system to $V_{\text {limit }}=24$ might achieve the Spaceguard goal and even more (if it were commissioned yesterday) by eventually pushing the $90 \%$ completeness level to asteroids with $H \leq 20.5$. Less expensive options (e.g., a network of systems capable of achieving $V_{\text {limit }} \sim$ 21.5 or fainter) should be able to achieve $90 \%$ completion on $H \leq 18$ NEOs in a reasonable time frame. Development of a southern hemisphere NEO surveying system would 
decrease the time to achieve $90 \%$ completeness but this reduction in time needs to be balanced by the increase in costs. Of course, these conclusions rest on the validity of our NEO model and survey simulator, which could be further tested by extensive modeling of search programs with well-determined efficiency, accurately known pointing history, and good records of both discovered and redetected NEOs.

This study, and the detailed analyses of others, support the superiority of space-based systems for NEO surveying compared to Earth-based systems-especially in the detection of Atens and IEOs. But the decision on which direction to pursue, or the amount of effort and money to direct in each, should depend on their relative merit in terms of cost per identified dangerous NEO and the time required to reduce the impact risk to an acceptable background level.

\section{Acknowledgments}

We thank Bob McMillan for valuable discussions and input to this study. Grant Stokes and another anonymous reviewer offered valuable suggestions to improve the content and presentation. Part of this research was conducted using the resources of the Cornell Theory Center, which receives funding from Cornell University, New York State, federal agencies, and corporate partners. Research funds were provided by NASA Grants NAGW-310 and NAG59082 and ESA Contract 14018/2000/F/TB. Travel support was provided by grants from NATO and NSF/CNRS. R. Jedicke is supported by grants to the Spacewatch project from NASA (NAG5-10447, NAG5-11828), AFOSR (F49620-00-1-0126), The Steven and Michele Kirsch Foundation, The Paul G. Allen Charitable Foundation, and other contributors.

\section{References}

Astronomy and Astrophysics in the New Millenium, 2000. p. 69. National Academy Press, Washington, D.C. Available at: http://books.nap.edu/ books/0309070317/html/R1.html.

Bottke, W.F., Jedicke, R., Morbidelli, A., Petit, J.-M., Gladman, B., 2000a Understanding the distribution of near-Earth asteroids. Science 288, 2190-2194.

Bottke, W.F., Morbidelli, A., Jedicke, R., Petit, J-M., Levison, H., Michel, P., Metcalfe, T., Gladman, B., 2001. Debiased orbital and size distributions of the near-Earth objects. Icarus 156, 399-433.

Bowell, E., Muinonen, K., 1994. Earth-crossing asteroids and comets: Groundbased search strategies. In: Gehrels, T., Matthews, M.S. (Eds.), Hazards Due to Comets and Asteroids. Univ. of Arizona Press, Tucson, pp. 417-462.
D’Abramo, G., Harris, A.W., Boattini, A., Valsecchi, G.B., 2001. A simple probabilistic model to estimate the population of near-Earth asteroids. Icarus $153,214-217$.

Gladman, B.J., Migliorini, F., Morbidelli, A., Zappalà, V., Michel, P., Cellino, A., Froeschlé, C., Levison, H.F., Bailey, M., Duncan, M., 1997. Dynamical lifetimes of objects injected into asteroid belt resonances. Science 277, 197-201.

Harris, A.W., 1998. Evaluation of ground-based optical surveys for nearEarth asteroids. Planet. Space Sci. 46, 283-290.

Helin, E.F., Shoemaker, E.M., 1979. Palomar planet crossing asteroid survey. Icarus $31,415-419$.

Jedicke, R., 1996. Detection of near Earth asteroids based upon their rates of motion. Astron. J. 111, 970

Jedicke, R., Herron, J.D., 1997. Observational constraints on the Centaur population. Icarus $127,494-507$.

Jedicke, R., Metcalfe, T.S., 1998. The orbital and absolute magnitude distributions of main belt asteroids. Icarus 131, 245-260.

Kresak, L., 1979. Dynamical interrelations among comets and asteroids. In: Gehrels, T. (Ed.), Asteroids. Univ. of Arizona Press, Tucson, pp. 289-309.

Levison, H.F. 1996. Comet taxonomy. In: Completing the Inventory of the Solar System, ASP Conf. Ser. 107, pp. 173-191.

Levison, H.F., Morbidelli, A., Dones, L., Jedicke, R., Wiegert, P.A., and Bottke, W.F., 2002. The mass disruption of Oort cloud comets. Science 296, 2212.

Mignard, F., 2001a. Detection of Near Earth Objects with GAIA. Observatoire de la Cote d'Azur/CERGA, SAG_FM_007, Release 1.

Mignard, F., 2001b. Phase Effect in the Detection of NEOs with GAIA. Observatoire de la Cote d'Azur/CERGA, SAG_FM 008.

Morbidelli, A., Jedicke, R., Bottke, W.F., Michel, P., Tedesco, E.F., 2002. From magnitudes to diameters: The albedo distribution of near Earth objects and the Earth collision hazard. Icarus 158, 329-342.

Morrison, D., 1992. The Spaceguard survey: Report of the NASA International Near-Earth-Object Detection Workshop (David Morrison, Chair). Jet Propulsion Laboratory/California Institute of Technology, Pasadena, CA.

Muinonen, K., 1998. Discovery and follow-up simulations for small Earthcrossing asteroids. Planet. Space Sci. 46, 291-297.

Rabinowitz, D.L., 1991. Detection of Earth-approaching asteroids in near real time. Astron. J. 101 (4), 1518-1529.

Rabinowitz, D., Bowell, E., Shoemaker, E.M., Muinonen, K., 1994. The population of Earth-crossing asteroids. In: Gehrels, T., Matthews, M.S. (Eds.), Hazards Due to Comets and Asteroids. Univ. of Arizona Press, Tucson, pp. 285-312.

Rabinowitz, D.L., Helin, E., Lawrence, K., Pravdo, S., 2000. A reduced estimate of the number of kilometre-sized near-Earth asteroids. Nature 403, 165-166.

Report of the Task Force on Potentially Hazardous Near Earth Objects 2000. British National Space Centre, London. Available at: http:// www.nearearthobjects.co.uk/downloads/full_report.pdf

Stokes, G.H., Evans, J.B., Viggh, H.E.M., Shelly, F.C., Pearce, E.C., 2000. Lincoln near-Earth asteroid program (LINEAR). Icarus 148, 21-28.

Stuart, J.S., 2001. A near-Earth asteroid population estimate from the LINEAR survey. Science 294, 1691.

Tedesco, E.F., Muinonen, K., Price, S., 2000. Space-based infrared nearEarth asteroid survey simulation. Planet. Space Sci. 48, 801-816.

Wetherill, G.W., 1967. Collisions in the asteroid belt. J. Geophys. Res. 72 (9), 2429 